\title{
Causes of non-purulent pleural effusions in a region with high prevalence of tuberculosis and HIV infections
}

\author{
${ }^{1}$ Ekpe E. E, ${ }^{2}$ Obot V. \\ ${ }^{I}$ Cardiothoracic Surgery unit, Department of Surgery, University of Uyo Teaching Hospital, P.M.B. 1136, Uyo, \\ Akwa Ibom State, Nigeria \\ ${ }^{2}$ Directorate of Public Health, Ministry of Health, Uyo, Akwa Ibom, Nigeria
}

\begin{abstract}
Background: Pleural effusion being excessive accumulation of fluid in the pleural cavity can occur as a result of lung, pleural or systemic disorders. For appropriate treatment, the specific cause must be known. Patients and methods: Retrospective chart analysis of 105 patients with non purulent pleural effusion was done for demography, symptoms, pleural fluid biochemical, microbiological, cytological, and pleural biopsy studies. Results: Of the 105 patients studied, there were 50 males and 55 females (M: $F=1: 1.1)$ with age from six months to 69 years and mean $37.8( \pm 11.5)$. The most common symptoms were difficulty in breathing, cough and chest pain which were complained of by 100\%, $97.1 \%$ and $92.4 \%$ of the patients. The pleural fluid was serous in about $75 \%$ (79 patients) and haemorrhagic in 26 patients (25\%). Biochemical quantitative analysis of the pleural fluids for protein and lactate dehydrogenase differentiated the pleural effusion into 12 transudative and 93 exudative pleural effusions based on cut-off pleural fluid protein of $30 \mathrm{~g} / \mathrm{L}$ and $\mathrm{LDH}$ of $200 \mathrm{U} / \mathrm{L}$. The transudative pleural effusions were diagnosed in nine of the 10 patients with congestive heart failure while the remaining three patients with transudative pleural effusions had amoebic liver abscess with sterile sympathetic right pleural effusion (two cases) and one case of chronic renal failure. Gram stains were suggestive of infection in 21 (20\%) patients while cultures confirmed the infection in only nine (42.8\%) of the 21 patients. Pneumonia was the most common cause of non purulent pleural effusion accounting for $31.4 \%$ of the cases while tuberculosis ranked second with 28.6\%. Breast cancers (all in women), congestive heart failure, urogenital cancers, and lung cancers accounted for 10.5\%, 9.5\%, $6.7 \%$ and $4.8 \%$ respectively.
\end{abstract}

Conclusion: This study shows that pneumonia and tuberculosis are the two most common causes of non purulent pleural effusions in the region.

Keywords: Pleural effusion, Tuberculosis, HIV infection

\section{Introduction:}

A pleural effusion is an excessive accumulation of fluid in the pleural space because of disorders of the lung or pleura, or to a systemic disorder. ${ }^{1}$ It is important to determine the aetiology of pleural effusion in order to treat it appropriately. Although in about $20 \%$ the aetiology maybe undetermined. ${ }^{1}$ Diagnostic evaluation of non purulent pleural effusion include biochemical and microbiological studies, cytological analysis and Immunohisto chemistry.

A useful approach to the diagnosis of pleural effusion is to initially differentiate the pleural effusion into transudate and exudate according to the Light's criteria. ${ }^{2,3}$ A transudative pleural effusion develops when the systemic factors influencing the formation or absorption of the pleural fluid are altered. The pleural surfaces are not involved by the primary pathologic process. Among the conditions that produce transudative pleural effusion, congestive heart failure is by far the most common. ${ }^{4}$ Pulmonary embolism, cirrhosis of the liver with ascites, and the nephrotic syndrome are the other common causes of transudative pleural effusion. ${ }^{4}$ It is generally known that benign pleural effusions are twice as common as malignant pleural effusion among the exudative pleural effusions. ${ }^{5}$ Pneumonia, cancer, tuberculosis, and pulmonary embolism account for most exudative effusions. Many pleural fluid tests are useful in the differential diagnosis of exudative effusions. Other tests helpful for diagnosis include helical computed tomography and thoracoscopy. ${ }^{3}$ The useful pleural fluid diagnostic tests include protein and albumin estimation, glucose, $\mathrm{pH}$, lactate dehydrogenase, amylase, adenosine deaminase, interferon-gamma, total white cell count and differential, packed cell volume, gram stain, microscopy, culture and sensitivity, acid fast bacilli stain and mycobacterial culture, and cytology. ${ }^{3,6,7} \mathrm{~A}$ thorough diagnostic work-up is necessary since an appropriate and effective treatment depend on the aetiology of the pleural effusion in the patient.

Studies have shown differences in aetiologies of pleural effusion in regions with high and low prevalence of tuberculosis and human immune deficiency virus infection. ${ }^{8,9}$ 
Since Nigeria still has a high burden of tuberculosis and HIV infection with estimated prevalence of 270 per 100,000 persons and $4.4 \%$ respectively, ${ }^{10,11}$ this study attempts to discover the causes of non purulent pleural effusion in one tertiary health institution in the country.

\section{Patients and methods}

We retrospectively analyzed the medical records of 105 patients with non purulent pleural effusion who presented from January 2007 to December 2011 (5 years) at University of Uyo Teaching Hospital, a tertiary referred medical centre in southern Nigeria. Non purulent pleural effusion was defined as pleural effusion that was not grossly purulent fluid; but may have positive effusion culture; and positive Gram's stain for bacteria.

The following data were collected for each patient: age, gender, clinical symptoms, important underlying diseases, leukocyte count, and duration of preadmission symptoms. The characteristics of pleural effusion, including gross appearance, cell count, $\mathrm{pH}$, glucose, protein, LDH, Gram's stain, acid-fast stain, culture findings and cytology; and percutaneous pleural biopsy histology results were also recorded.

Patients who had incomplete documentation of their medical records were excluded from the study. So also were patients with purulent pleural effusion on gross examination of the sampled pleural fluid.

Analysis was by comparative proportions and percentages.

\section{Results}

A total of 105 patients with non purulent pleural effusion who presented during the study period met the inclusion criteria. There were 50 males and 55 females (M: $F=1: 1.1)$ with age range from six months to 69 years and mean 37.8 ( \pm 11.5 ) (table 1$)$. The most common symptoms amongst the 105 patients were difficulty in breathing, cough and chest pain which were complained of by $100 \%, 97.1 \%$ and $92.4 \%$ of the patients respectively, while fever was complained of by about $50 \%$, coughing out of blood by about $16 \%$, weight loss by about $54 \%$ and drenching night sweats by $28 \%$. (table 2). All the patients had their pleural effusion confirmed by plain chest radiographs, while ultrasonography was used in patients who had additional indication for ultrasound scanning including intra-abdominal pathology and staging for malignancy. Chest computed tomographic scan was utilized in some of the patients with clinical diagnosis of malignant pleural effusion.

Diagnostic thoracocentesis was done on all the 105 patients for pleural fluid sampling for analysis as shown in table 3. The pleural fluid was serous in about $75 \%$ (79 patients) and haemorrhagic in 26 patients (25\%). Biochemical quantitative analysis of the 105 pleural fluids for protein and lactate dehydrogenase differentiated the pleural effusion into 12 transudative and 93 exudative pleural effusions based on cut-off pleural fluid protein of $30 \mathrm{~g} / \mathrm{L}$ and $\mathrm{LDH}$ of $200 \mathrm{U} / \mathrm{L}$. The transudative pleural effusions were diagnosed in nine of the 10 patients with congestive heart failure while the remaining three patients with transudative pleural effusions had amoebic liver abscess with sterile sympathetic right pleural effusion (two cases) and one case of chronic renal failure.

All the patients with haemorrhagic pleural effusion were diagnosed as having malignant pleural effusion and packed cell volume (PCV) estimation of the pleural fluid was less than 50\% of the peripheral blood PCV which excluded the diagnosis of haemothorax. Further analysis shows that cytological analysis of the fluid revealed the presence of malignant cells in 14 (about 54\%) of the 26 patients with malignant pleural effusion, while percutaneous pleural biopsy in the same sub-group of patients revealed malignant histology in 19 (73\%)of the 26 patients.

Amongst the patients with non purulent pleural effusions Gram stains were suggestive of infection in $21(20 \%)$ patients while cultures confirmed the infection in only nine $(42.8 \%)$ of the 21 patients. This disparity is thought to be as a result of widespread use of antibiotics both prescribed and unprescribed before presentation to hospital. All the exudative pleural effusions were characterized by markers of inflammatory response including low level of pleural fluid glucose below 40mg.dL (about 89\%), low pleural fluid pH less than 7.30 (about $88 \%$ ), and high pleural fluid total white cell count exceeding $6.0 \times 10^{9} / \mathrm{L}$ (about $88 \%$ ). Pleural fluid Ziehl-Nelsen stain for acid fast bacilli was positive in only 14 (about $47 \%$ ) of the 30 patients with diagnosis of tuberculosis. The HIV infectivity rate amongst the 105 patients with non purulent pleural effusion studied was $19 \%$.

Table 4 shows the aetiologies of non purulent pleural effusion in the 105 patients.

Pneumonia was the most common cause of non purulent pleural effusion accounting for $31.4 \%$ of the cases while tuberculosis ranked second with $28.6 \%$. Breast cancers (all in women), congestive heart failure, urogenital cancers (including metastatic prostate cancer, bladder cancer and renal cell carcinoma), and lung cancers accounted for $10.5 \%, 9.5 \%, 6.7 \%$ and $4.8 \%$ respectively. The remaining nine $(8.6 \%)$ cases of non purulent pleural effusion in this series were caused by one case each of metastatic soft tissue sarcoma, primary liver cell carcinoma, malignant mediastinal tumour, and mesothelioma; two cases of sympathetic pleural effusion secondary to amoebic liver abscess; and one case each of thoracic endometriosis syndrome, rheumatoid arthritis, and chronic renal failure. 


\section{Discussion:}

There are many causes of non purulent pleural effusion which range from diseases of the lung or pleura that cause exudative pleural effusion to systemic diseases that cause transudative pleural effusion. The present study shows that the non purulent pleural effusion can occur in all age groups from infancy to elderly. The youngest patient in our series aged six months while the oldest aged 69 years. Other related studies have also shown that no age group is immune to this problem. ${ }^{8,12}$ However the median age of the patients occurs in the productive age where loss of one man-hour means a lot to the economic output of the society. Various other studies have documented varying sex ratio of incidence of pleural effusion, ${ }^{8,12,13}$ which in this study stands at male to female ratio of $1: 1.1$. The higher female incidence is contributed to by malignant pleural effusions caused by advanced breast cancers which were noted in women only.

Respiratory symptoms alone are inadequate in identification of the cause of non purulent pleural effusion. However combination of symptoms and clinical signs would in most cases be sufficient for the diagnosis of pleural effusion, while the addition of laboratory investigations will enable the identification of the cause of the pleural effusion which is necessary for appropriate treatment. ${ }^{3}$ In the present study difficulty in breathing was experienced by $100 \%$ of the patients which is to some extent a reflection of the degree of lung compression caused by the pleural effusion. The second most common symptom amongst the 105 patients was cough which was present in about $97 \%$ of the patients. In more than half of the patients experiencing cough, there was production of sputum and in about $16 \%$ of the patients there was haemoptysis. Chest pain was present in $92.4 \%$ of the patients with non purulent pleural effusion. The other symptoms encountered in the series were involuntary weight loss $(54.3 \%)$ in the subset of the patients with tuberculosis or cancer; fever $(50.5 \%)$ objectively confirmed or described as "internal heat" unable to be confirmed clinically. It was only the subset of the patients with pulmonary tuberculosis $(28.6 \%)$ that experienced drenching night sweats. In the study by Gopi et al, chest pain and cough were the most prominent symptoms which occurred in $75 \%$ and $70 \%$ of the patients respectively. ${ }^{14}$

Pleural fluid analysis, cytology and pleural biopsy histology were useful in elucidating the causes of non purulent pleural effusion in this study. Macroscopic appearance enabled patients with purulent pleural effusion (empyema thoracis) to be excluded from the study. Analyses for protein and lactate dehydrogenase successfully enabled the characterisation of the pleural effusion as exudates $(88.6 \%)$ and transudate $(11.4 \%)$. All the transudates were in nine of the 10 patients with congestive heart failure, the two patients with sympathetic pleural effusion and one patient with chronic renal failure. In the study by Zablockis et al, transudate accounted for $24 \%$ of pleural effusion and were caused by congestive cardiac failure, nephrotic syndrome and liver cirrhosis, while exudates accounted for $76 \% .^{6}$ Other investigations carried out on the pleural fluid gave results that corroborated previous studies. ${ }^{715}$ These include glucose level and $\mathrm{pH}$ of the exudative pleural fluid which showed consistent low values $(<2.2 \mathrm{mmol} / \mathrm{L}$ and $<7.30$ respectively), and high total white cell count $\left(>6.0 \times 10^{9} / \mathrm{L}\right)$ in patients with pleural sepsis. Also packed cell volume was greater than $1.0 \%$ but $<10 \%$ in the 26 patients with haemorrhagic pleural effusion. Out of the 21 patients shown by positive gram stain to have infective pleural effusion, culture confirmation was positive in only nine patients. This high negative culture rate is attributed to abuse of antibiotics in the country where almost all antibiotics can be purchased as on-thecounter drugs. Previous study had shown this situation to favour progression to empyema thoracis amongst children with bronchopneumonia. ${ }^{16}$ Again of the 30 patients with tuberculosis, acid fast bacilli were seen in the pleural fluid of 14 patients. Similar finding has been made by similar studies and explained by the fact that most pleural effusions in tuberculosis develop as a result of hypersensitivity reaction to the mycobacterial antigen in the pleural membranes. ${ }^{17}$ For this reason additional diagnostic benefit is obtained by quantitative estimation of pleural fluid adenosine deaminase (ADA) of above 40U/L and interferon - gamma (INF- $\gamma$ ) above 3.7U/L and pleural fluid mycobacerial culture using Lowenstein-Jensen or BACTEC culture medium. ${ }^{17}$ Non of the above listed tests was available in our institution for use in the index study. Blind percutaneous pleural biopsy for histology using Abram's needle showed a higher positivity rate compared to pleural fluid cytology (70.4\% versus $51.9 \%$ ). The positivity of pleural biopsy can be further enhanced by thoracoscopy which directs tissue biopsy to area of the pleura with suspicious appearance. Similar results have been found by other relatedstudies including that of Light which gave cytology positivity of 40-77\% against pleural biopsy positivity of 50-80\% in tuberculosis and $37-75 \%$ in malignancy, which is further increased to $95-100 \%$ when the biopsy is obtained via thoracoscopy. ${ }^{15}$

This study conducted in a region of high prevalence of tuberculosis and HIV infections has shown HIV infection rate of $19 \%$ among patients with non purulent pleural effusion and that benign pleural effusion occurs more frequently than malignant pleural effusion (75\% versus $25 \%$ ), with $19 \%$ of patients with non purulent pleural effusion being HIV positive compared to the $40.9 \%$ in the Owino, et al study which also showed that 
$42.3 \%$ of the patients with tuberculous pleural effusion were HIV positive. ${ }^{12}$ Thomas, et al also noted that benign pleural effusions were twice as common as malignant pleural effusions. ${ }^{5}$ This study documents parapneumonic pleural effusion in $42 \%$ of the patients. Parapneumonic pleural effusion has been noted to occur in up to $40 \%$ of all pneumonia. ${ }^{13}$ Among the benign causes of non purulent pleural effusion in this study, tuberculosis is only second to pneumonia (38\% versus $42 \%$ ). Tuberculous pleural effusion which has been studied extensively occurs in all age groups and in both males and females and accounts for about $44 \%$ of pleural effusion in regions with high prevalence of the disease where it is also commoner in younger patients. ${ }^{8}$ The study by Vaides et al found tuberculous effusion to account for $25 \%$ of the 642 patients with pleural effusion, more on the right side and occurring in up to $30 \%$ of tuberculosis patients ${ }^{13}$ With the depression of immunity which occurs with HIV infection, latent tuberculosis including tuberculous pleurisy which commonly present with pleural effusion gets reactivated. This explains why tuberculosis is responsible for up to $38 \%$ of non purulent pleural effusion in this study. Congestive heart failure was responsible for $12.8 \%$ of the benign pleural effusion in this study. Expectantly all but one of the CHF associated pleural effusions were transudate. ${ }^{18,19}$ The only exudative non purulent pleural effusion in a patient with CHF was unilateral, associated with fever, and therefore thought to be probably a parapneumonic pleural effusion occurring in a patient with congestive heart failure although the white cell count of the pleural fluid was less than $6.0 \times 10^{9} / \mathrm{L}$. The remaining benign causes of non purulent pleural effusion in this series were two cases of sterile sympathetic pleural effusion occurring in two patients with amoebic liver abscess, and one case each caused by rheumatoid arthritis, thoracic endometriosis syndrome and chronic renal failure. These causes have variedly been discovered as causes of pleural effusion in other studies. ${ }^{7}$

Malignancy accounted for $25.7 \%$ of the causes of non purulent pleural effusion in this study and is about three times less common than benign causes. An approximate ratio was found by Liam et al who found malignant pleural effusion in less than $30 \%$ of their 186 patients with exudative pleural effusion. ${ }^{8}$ Breast cancer all occurring in women accounted for $40.7 \%$ of the malignant causes of non purulent pleural effusion in this study. This proportion was higher than that found in the study by Zarogoulidis et al where breast cancer accounted for only $25 \%$ of malignant pleural effusion while lung cancer accounted for $40 \%{ }^{20}$ In this study lung cancer accounted for only $18.5 \%$ of malignant pleural effusion which may actually mean under-diagnosis since a recent study has discovered an increasing incidence of lung cancers in the country. ${ }^{21}$ Palliative treatment was adopted in all cases of malignant pleural effusion. ${ }^{22}$

\section{Conclusion:}

This study conducted in a region with high prevalence of tuberculosis and H.I.V. infections shows that pneumonia and tuberculosis are the two most common causes of non purulent pleural effusions. Prompt diagnosis, case finding, contact tracing and appropriate treatment of pneumonia and tuberculosis in the region would go a long way to reducing the incidence of non purulent pleural effusions.

Conflict of interest: "The author(s) declare(s) that there is no conflict of interests regarding the publication of this article."

\section{References:}

[1]. Karkhanis VS, Joshi IM. Pleural effusion: diagnosis, treatment, and management. Open Access Emergency Medicine 2012:4 31-52

[2]. Light RW. Management of pleural effusions. J Formos Med Assoc. 2000 Jul;99(7):523-31.

[3]. Porcel JM, Light RW. Diagnostic approach to pleural effusion in adults. Am Fam Physician. 2006 Apr 1;73(7):1211-20.

[4]. Chetty KG. Transudative pleural effusions. Clin Chest Med. 1985 Mar;6(1):49-54.

[5]. Thomas R, Lee YC. Causes and management of common benign pleural effusions. Thorac Surg Clin. 2013 Feb;23(1):25-42, v-vi. doi: 10.1016/j.thorsurg.2012.10.004.

[6]. Zablockis R, Nargela R. Diagnostic value of pleural fluid cytologic examination. Medicina (Kaunas). 2002;38(12):1171-8.

[7]. BTS guidelines for the investigation of a unilateral pleural effusion in adults. Thorax 2003;58:ii8-ii17 doi:10.1136/thorax.58.suppl_2.ii8

[8]. Liam CK, Lim KH, Wong CM. Causes of pleural exudates in a region with a high incidence of tuberculosis. Respirology. 2000 Mar;5(1):33-8.

[9]. Richter C, Perenboom R, Swai AB, Kitinya J, Mtoni I, Chande H, Kazema RR, Chuwa LM, Mwakyusa DH, Maselle SY. Diagnosis of tuberculosis in patients with pleural effusion in an area of HIV infection and limited diagnostic facilities. Trop Geogr Med. 1994;46(5):293-7.

[10]. http://www.tradingeconomics.com/nigeria/incidence-of-tuberculosis-per-100-000-people-wb-data.html

[11]. http://www.nigeriahivinfo.com/

[12]. Owino EA, McLigeyo SO, Gathua SN, Nyong'o A. Prevalence of human immunodeficiency virus infection: its impact on the diagnostic yields in exudative pleural effusions at the Kenyatta National Hospital, Nairobi. East Afr Med J. 1996 Sep;73(9):575-8.

[13]. Vaides L, Alvarez D, Voile M, Pose A, San Jose E. The Etiology of Pleural Effusions in an Area With High Incidence of Tuberculosis. CHEST 1996;109(1):158-162

[14]. Gopi A, Madhavan SM, Sharma SK, Sahn SA. Diagnosis and Treatment of Tuberculous Pleural Effusion in 2006. CHEST 2007; 131(3):880-9

[15]. Light RW. Diagnostic principles in pleural disease. Eur Respir J 1997; 10: 476-481

[16]. Ekpe EE. Akpan MU. Poorly treated broncho-pneumonia with progression to empyema thoracis in Nigerian children. TAF Preventive Medicine Bulletin 2010;9(3):181-186 
[17]. Porcel JM. Tuberculous pleural effusion. Lung. 2009 Sep-Oct;187(5):263-70. doi: 10.1007/s00408-009-9165-3. Epub 2009 Aug 13.

[18]. Chetty KG. Transudative pleural effusions. Clin Chest Med. 1985 Mar;6(1):49-54.

[19]. Rubins JB, Colice GL. Evaluating pleural effusions. How should you go about finding the cause? Postgrad Med. 1999 May 1;105(5):39-42, 45-8.

[20]. Zarogoulidis K, Zarogoulidis P, Darwiche K, Tsakiridis K, Machairiotis N, Kougioumtzi I, Courcoutsakis N, Terzi E, Zaric B, Huang H, Freitag L, Spyratos D. Malignant pleural effusion and algorithm management. J Thorac Dis. 2013 September; 5(Suppl 4): S413-S419. doi: 10.3978/j.issn.2072-1439.2013.09.04

[21]. Ezemba N., Ekpe E.E., Eze J.C. Challenges of lung cancer management in a developing Country. Nig J.M. 2012; 21: 214-217

[22]. Ekpe EE, Ikpe MC, Umanah IN. Palliative treatment of malignant pleural effusion.Diagnostic and Therapeutic Study 2013; 2: 8 -20.

VI. Tables of Results

Table 1: Age/Sex Distribution

\begin{tabular}{|l|l|l|l|}
\hline AGE (YEARS) & MALE $(\%)$ & FEMALE $(\%)$ & TOTAL $(\%)$ \\
\hline$<1$ & $2(1.9)$ & $0(0)$ & $2(1.9)$ \\
\hline $1-10$ & $2(1.9)$ & $2(1.9)$ & $4(3.8)$ \\
\hline $11-20$ & $4(3.8)$ & $4(3.8)$ & $8(7.6)$ \\
\hline $21-30$ & $10(9.5)$ & $7(6.7)$ & $17(16.2)$ \\
\hline $31-40$ & $9(8.6)$ & $16(15.2)$ & $25(23.8)$ \\
\hline $41-50$ & $9(8.6)$ & $11(10.5)$ & $20(19.0)$ \\
\hline $51-60$ & $6(5.7)$ & $9(8.6)$ & $15(14.3)$ \\
\hline$>60$ & $8(7.6)$ & $6(5.7)$ & $14(13.3)$ \\
\hline TOTAL & $50(47.6)$ & $55(52.4)$ & $105(100)$ \\
\hline
\end{tabular}

Table 2: Presenting symptoms among 195 patients with non purulent pleural effusion

\begin{tabular}{|l|l|l|}
\hline Presentation & No of Pts & $\%$ \\
\hline Difficulty in breathing & 105 & 100 \\
\hline Fever/ 'internal heat' & 53 & 50.5 \\
\hline Weight loss & 57 & 54.3 \\
\hline Night sweat & 30 & 28.6 \\
\hline Cough & 102 & 97.1 \\
\hline Coughing out blood & 17 & 16.2 \\
\hline Chest pain & 97 & 92.4 \\
\hline
\end{tabular}

Table 3: Characteristics of Pleural Fluid of 105 Patients with Non Purulent Pleural Effusion

\begin{tabular}{|c|c|c|}
\hline VARIABLES & FREQUENCY $(\%)$ & TOTAL (\%) \\
\hline Haemorrhagic & $26(24.8)$ & 105 \\
\hline$<30$ & $12(11.4)$ & \multirow[t]{2}{*}{105} \\
\hline$>30$ & $93(88.6)$ & \\
\hline \multicolumn{3}{|c|}{ GLUCOSE (g/dL) } \\
\hline \multicolumn{3}{|l|}{$\mathrm{pH}$} \\
\hline$<7.30$ & $92(87.6)$ & \multirow[t]{2}{*}{105} \\
\hline$>7.30$ & $13(12.4)$ & \\
\hline \multicolumn{3}{|c|}{ WHITE BLOOD CELLS COUNT (X10 $/ \mathrm{L})$} \\
\hline$<6.0$ & $12(11.4)$ & 105 \\
\hline \multicolumn{3}{|c|}{ LACTATE DEHYDROGENASE (U/L) } \\
\hline$<200$ & $12(11.4)$ & \multirow[t]{2}{*}{105} \\
\hline$>200$ & $93(88.8)$ & \\
\hline GRAM STAIN & & \\
\hline
\end{tabular}


Causes Of Non-Purulent Pleural Effusions In A Region With High Prevalence Of Tuberculosis..

\begin{tabular}{|l|l|l|}
\hline Positive & $21(20.0)$ & 105 \\
\hline Negative & $84(80.0)$ & \\
\hline CULTURE & & 105 \\
\hline Positive & $9(8.5)$ & \\
\hline Negative & $96(91.5)$ & 105 \\
\hline SEROLOGY & & \\
\hline Reactive & $20(19.0)$ & 105 \\
\hline Non-reactive & $85(81.0)$ & \\
\hline ACID FAST BACILLI & & \\
\hline Seen & $14(13.3)$ & 105 \\
\hline Not seen & $91(86.7)$ & \\
\hline CYTOLOGY & & \\
\hline Positive for malignant cells & $14(13.3)$ & \\
\hline Negative for malignant cells & $91(86.7)$ & 105 \\
\hline PLEURAL BIOPSY & & \\
\hline Malignant histology & $19(18.1)$ & \\
\hline Benign histology & $86(81.9)$ & \\
\hline
\end{tabular}

Table 4: Causes Of Non Purulent Pleural Effusion In 105 Patients In A Region With High Prevalence Of Tb And Niv Infections

\begin{tabular}{|l|l|l|l|}
\hline CAUSE & MALE $(\%)$ & FEMALE $(\%)$ & TOTAL $(\%)$ \\
\hline Pneumonia & $15(14.3)$ & $18(17.1)$ & $33(31.4)$ \\
\hline Pleuropulmonary tuberculosis & $18(17.1)$ & $12(11.4)$ & $30(28.6)$ \\
\hline Breast cancer & $0(0)$ & $11(10.5)$ & $11(10.5)$ \\
\hline Cardiac & $6(5.7)$ & $4(3.8)$ & $10(9.5)$ \\
\hline Urogenital cancers & $4(3.8)$ & $3(2.9)$ & $7(6.7)$ \\
\hline Lung cancer & $3(2.9)$ & $2(1.9)$ & $5(4.8)$ \\
\hline Other cancers & $2(1.9)$ & $2(1.9)$ & $4(3.8)$ \\
\hline Sympathetic & $1(0.9)$ & $1(0.9)$ & $2(1.9)$ \\
\hline Others & $1(0.9)$ & $2(1.9)$ & $3(2.9)$ \\
\hline Total & $50(47.6)$ & $55(52.4)$ & $105(100)$ \\
\hline
\end{tabular}

